\section{Danger to science of no-deal Brexit}

\section{As UK-based European} stakeholders, we are deeply concerned about the threat that Brexit - particularly a 'no deal' scenario - poses to international research (Nature 572, 13-14; 2019).

Uncertainties arising from the 2016 Brexit referendum have already undermined the attraction for foreigners of doing research in Britain. In our view, the various scenarios are all likely to damage research initiatives.

'Shadow membership' and 'third country' scenarios, for example, represent different degrees of cooperation with the European Union. These could introduce new challenges, and perhaps opportunities, with regard to partnerships, taxes and regulations. But they would still curtail the freedom enjoyed by European academics. The UK government would need to increase its research budget to offset the loss of the EU funding.

Scientific excellence is underpinned by researcher mobility, adequate resources and regulations that foster longterm stability and planning. A no-deal scenario would result in fewer European collaborations, diminished resources and constrained legal frameworks. It would therefore present a grave danger to science.

Mariana Pinto da Costa ${ }^{\star}$ Queen Mary University of London, UK. mariana.pintodacosta@qmul.ac.uk ${ }^{*}$ On behalf of 4 correspondents; see go.nature.com/33rpv9j.

\section{Astronomy's ethical duty to Hawaiian site}

As a partner of the Thirty Meter Telescope (TMT) Consortium and a member of Qalipu Mi'kmaq First Nations, I am one of very few Indigenous faculty members in Canadian astronomy. In my view, Canada's astronomy community has an ethical duty to listen to the Native Hawaiian protectors of the sacred Mauna Kea site, where the consortium now has a permit for construction. Our response will affect the future of astronomy and reconciliation with Indigenous peoples.

According to the United Nations Declaration on the Rights of Indigenous Peoples (UNDRIP) and the Calls of Action of the Truth and Reconciliation Commission of Canada, Canada and the TMT consortium have a duty to respect the wishes of the protectors, along with Indigenous peoples' rights, wherever we pursue astronomical discovery. The astronomy community should therefore halt construction, listen to the protectors and support those protesters who have been arrested.

If the consortium is not willing to step back, then Canada must remove itself from the project as part of its commitment to UNDRIP. Otherwise, we continue to support a culture that does not respect the right of self-determination and is not inclusive of Indigenous peoples. Hilding Neilson University of Toronto, Canada.

hilding.neilson@utoronto.ca

\section{Sand: an overlooked occupational hazard}

Mette Bendixen and colleagues point out the environmental, social and economic harms that sand extraction might cause (Nature 571, 29-31; 2019). It can also affect human health, a particularly important point for workers. A global agenda for sustainable sand extraction should incorporate workers' health policies to prevent silicosis and other serious lung diseases.

The surface properties that make sand from deserts or beaches unsuitable for the building industry also make it less hazardous when inhaled by humans. However, long-term inhalation of small crystalline particles of silica (sand's primary component) can lead not just to silicosis, a progressive and incurable fibrotic lung disease, but to lung cancer, chronic obstructive pulmonary disease, autoimmune disease and tuberculosis (P. Cullinan et al. Lancet Respir. Med. 5, 445; 2017). Hazardous jobs that involve exposure to freshly fractured silica include crushing, milling, processing, drilling, grinding, polishing and cutting materials containing quartz. Silicosis remains a public-health problem in emerging economies.

Regulations and strategies for controlling exposure have helped to reduce the incidence of silicosis in high-income countries. However, outbreaks among workers fabricating countertops from natural stone powders in resin binders demonstrate an unacceptable ignorance of this health hazard (Lancet Respir. Med. 7, 283; 2019). Steven Ronsmans, Benoit Nemery Centre for Environment and Health, Leuven, Belgium. steven.ronsmans@kuleuven.be

\section{Sand: save it for sea-level rise}

Mette Bendixen and colleagues point out that sand extracted from fluvial environments is being consumed faster than it is produced (Nature 571, 29-31; 2019) This has deep implications for managing flood risk in a changing climate.

Extracting sand or restricting its movement (such as through river damming) reduces sediment availability. This means that when large floods occur, insufficient sediment is deposited on the land for it to act as a defence against smaller floods. Fluvial-sediment depletion can also lead to coastal erosion, especially if accompanied by illegal sand mining on the foreshore.

Sea-level rise is projected to accelerate in the second half of this century. According to Bendixen and colleagues, sand prices could be exceptionally high by then. Instead of squandering sand, we need to save it. Sally Brown, Susan Hanson University of Southampton, UK. sb20@soton.ac.uk

\section{Rule out nepotism in psychology awards}

The payment of substantive fees to some psychologists who give talks on their own research has sparked concerns over conflicts of interest (COIs; Nature 571, $20-23 ; 2019)$. We cannot rule out the possibility that the handing out of academic awards and prizes in psychology by professional societies or associations might also be subject to COIs.

We scrutinized the websites of 58 psychology societies using a pre-registered protocol (A. H. Stoevenbelt et al. Preprint at https://psyarxiv.com/phyu3; 2019). Our aim was to determine whether we could exclude the possibility that any recipients of such awards were closely affiliated with individuals on the award committees - for example, as family members, collaborators, mentees or colleagues.

Most of the societies (72.4\%) failed to highlight any potential COIs in the committees responsible for selecting award winners. Less than half of them (44.8\%) published no COI regulations at all. And, of those that did, only half $(27.6 \%)$ explicitly mentioned avoiding COIs in choosing prizewinners.

We urge psychology societies to avoid conveying the impression of hidden nepotism by openly publishing their policies on personal COIs. Andrea H. Stoevenbelt Tilburg University, the Netherlands. a.h.stoevenbelt@uvt.nl ${ }^{*}$ On behalf of 4 correspondents; see go.nature.com/2zj9y5k.

\section{CORRECTION}

In the Nature Index 2019

Annual Tables (Nature 570, S1S6; 2019) the fractional counts, percentage changes and article counts used for the tables were incorrect, which affected the rankings of some institutions. The updated data, graphics and rankings can be found online at https://www.nature. com/collections/fbfjafhcbb. 